\title{
Head and Neck Cancer
}

\section{Comparison of Two Standard Treatment Approaches in Locoregionally Advanced Nasopharyngeal Carcinoma}

\author{
Issa Mohamad ${ }^{1}$ Fawzi Abu-Hijleh ${ }^{1}$ Ebrahim Mayta ${ }^{2}$ Taher Abu-Hejleh ${ }^{3}$ Wisam Al-Gargaz ${ }^{2}$ \\ Abdellatif Al Mousa ${ }^{1}$ Ramiz Abu-Hijlih ${ }^{1}$ Ali Hosni ${ }^{4}$
}

${ }^{1}$ Department of Radiation Oncology, King Hussein Cancer Center, Amman, Jordan

2 Department of Surgical Oncology, King Hussein Cancer Center, Amman, Jordan

${ }^{3}$ Division of Hematology/Oncology, Department of Internal Medicine, University of lowa Hospitals and Clinics, lowa City, lowa, Unites States

${ }^{4}$ Radiation Medicine Program, Princess Margaret Cancer Centre, University Health Network, University of Toronto, ON, Canada
Address for correspondence Issa Mohamad, MD, Department of Radiation Oncology, King Hussein Cancer Center, PO Box 1269, Amman 11941, Jordan (e-mail: imohamad@khcc.jo).

South Asian J Cancer 2022;11(3):223-228.

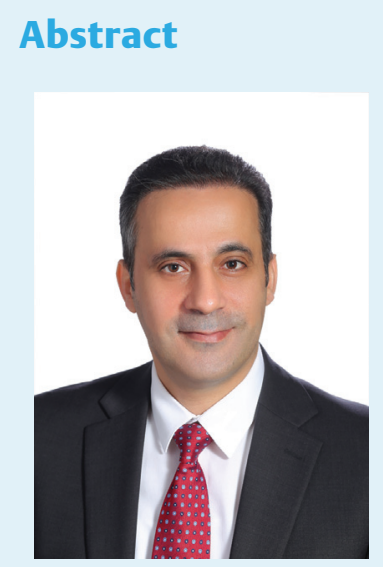

Issa Mohamad

Objectives To compare outcomes and toxicity of two standard treatment approaches of advanced nasopharyngeal carcinoma (NPC).

Methods Between 2010 and 2016, patients with NPC, stage II-IVa, treated with induction chemotherapy (IC) (TPF), followed by concurrent chemoradiotherapy (CCRT) (induction group), or CCRT, followed by adjuvant chemotherapy (AC) (PF) (no-induction group), were retrospectively reviewed. CCRT included platinum-based chemotherapy with intensity-modulated radiotherapy. Survival outcomes, the pattern of failures, toxicity, and predictors for survival outcomes were evaluated.

Results A total of 110 patients were included, 65 in the induction group and 45 in the no-induction group. There were no significant differences in the DFS and overall survival (OS) at 3 years between the two groups. On multivariate analysis, performance status ( 1 vs. 0 ) predicted worse OS. The 3 -year cumulative incidence rates for local, regional, and distant failures were 58.5\% (95\% confidence interval [CI]: 8.4-89\%), $58.00 \%$ (95\% Cl: $8-88.8 \%$ ), and 63.90\% (95\% Cl: $14.1-90.2 \%)$, respectively. IC had more frequent acute grade $(G)$ II anemia ( 13 vs. $1, p<0.01$ ), late $G$ II brain toxicity ( 4 vs. 1 , $p<0.01$ ), and late $G$ II dysphagia (32 vs. $11, p=0.01$ ).

Conclusions Survival outcomes were comparable between the two groups. IC had

Keywords

- nasopharyngeal carcinoma $\quad$ toxicity

- IMRT

- outcomes

- concurrent chemotherapy

DOI https://doi.org/10.1055/s-0042-1742724 ISSN 2278-330X

How to cite this article: Mohamad I, Abu-Hijleh F, Mayta E, et al. Comparison of Two Standard Treatment Approaches in Locoregionally Advanced Nasopharyngeal Carcinoma. South Asian J Cancer 2022;11(3):223-228. (c) 2022. Medlntel Services Pvt Ltd. All rights reserved.

This is an open access article published by Thieme under the terms of the Creative Commons Attribution-NonDerivative-NonCommercial-License, permitting copying and reproduction so long as the original work is given appropriate credit. Contents may not be used for commercial purposes, or adapted, remixed, transformed or built upon. (https://creativecommons.org/licenses/by-nc-nd/ 4.0/)

Thieme Medical and Scientific Publishers Pvt. Ltd., A-12, 2nd Floor, Sector 2, Noida-201301 UP, India 


\section{Introduction}

Nasopharyngeal carcinoma (NPC) differs from other cancers of the head and neck by epidemiology, histopathology, clinical characteristics, treatment outcomes, and patterns of failure. ${ }^{1}$ Radiation therapy constitutes the mainstay treatment with a different approach regarding the sequence of chemotherapy (i.e., induction versus adjuvant). ${ }^{2-7}$

In our region, clinical outcomes are derived from studies conducted in the endemic region. There are limited data on survival outcomes, patterns of failure, acute and late toxicity from the Middle East region. ${ }^{8,9}$ Herein, we retrospectively evaluated the clinical outcomes, patterns of failure, acute and late toxicities of NPC patients treated with CCRT with or without IC or AC at our institution.

\section{Materials and Methods}

\section{Eligibility Criteria}

From 2010 to 2016, after institutional research board (IRB) approval (IRB No. 14 KHCC 79), this retrospective study evaluated adult patients with non-metastatic, histologically confirmed NPC. The staging was reviewed according to the eighth edition of the TNM staging system jointly used by the American Joint Committee of Cancer and Union of International Cancer Control (AJCC/UICC). ${ }^{10}$ Patients were included if they had stage II-IVa and treated according to our institutional guidelines with either induction chemotherapy (IC) followed by IMRT-based concurrent chemoradiotherapy CCRT (induction group) or IMRT-based CCRT followed by adjuvant chemotherapy (AC) (no-induction group). Patients were excluded if they had stage I, second primary tumor, and previous history of radiotherapy or chemotherapy.

The patient's clinical and tumor characteristics are summarized in -Table 1. Patients had pre-treatment blood tests, hepatitis profile, cardiac evaluation, nasopharyngoscopy, head and neck magnetic resonance imaging (MRI), wholebody positron emission tomography (PET/CT) scan, and evaluated by supportive services.

The induction group received TPF (docetaxel $\left[75 \mathrm{mg} / \mathrm{m}^{2}\right]$ and cisplatin $\left[100 \mathrm{mg} / \mathrm{m}^{2}\right]$ on day 1 and 5 -fluorouracil [1000 $\mathrm{mg} / \mathrm{m}^{2}$ ] on days $1-5$ every 3 weeks for three cycles), followed by weekly carboplatin area under the curve (AUC) 1.5 during IMRT. The no-induction group received high dose cisplatin $100 \mathrm{mg} / \mathrm{m}^{2}$ on days 1,22 , and 43 during IMRT followed by PF (cisplatin $80 \mathrm{mg} / \mathrm{m}^{2}$ on day 1 and fluorouracil $1,000 \mathrm{mg} / \mathrm{m}^{2} / \mathrm{d}$ on days 1 to 4 was given every 4 weeks for three cycles). Carboplatin replaced cisplatin in concurrent or adjuvant settings if there was a contraindication for cisplatin.

IMRT was delivered in 33 to 35 fractions, 5 fractions per week over 6.5 to 7 weeks using a simultaneous-integrated boost technique (PTV1 received $70 \mathrm{~Gy} ; 33-35$ fraction and PTV2 received 54-56Gy in 33-35 fractions). An intermediate dose of 59.4-63 Gy was used in 33-35 fractions and allowed at the discretion of the treating radiation oncologist. Radiation therapy was delivered on a daily basis from Sunday to Thursday with daily bone match cone-beam CT (CBCT) image guidance.
All patients were followed up on a weekly basis during CCRT, then, at 3 month intervals or more frequently for the first 2 years and then at 4 - to 6-month intervals for the third year, and 6 to 12 months thereafter. Follow-up consisted of physical examination and endoscopic evaluation at each follow-up visit. Head and neck MRI was performed every 6 months for 2 years, and then annually or as clinically indicated.

Disease-free (DFS) and overall survival (OS) were analyzed using the Kaplan-Meier method and compared using the log-rank test. Local failure (LF), regional failure (RF), and distant failure (DF) rates were estimated using the cumulative incidence method, with death as a competing risk. Outcomes were calculated from the date of diagnosis to the first event. Acute and late toxicity rates were estimated using proportion and associated binomial 95\% confidence interval $(\mathrm{CI})$ and were compared between patients who received induction and no-induction using the Chi-square test. Multivariable analysis (MVA) using Cox proportional hazards regression was used to identify predictors of DFS and OS. All reported $p$-values were two-sided, with a statistical significance level of $p<0.05$. All analyses were performed using SAS version 9.4 (SAS Institute Inc, Cary, NC).

\section{Results}

Patient and tumor characteristics are summarized in -Table 1. For the entire cohort, the median age at diagnosis was 44 years (range: 18-76 years). The majority of patients were males (70.9\%), had undifferentiated nonkeratinizing NPC (89.1\%) and EBV was positive in (65.2\%) of tested patients. Patients were staged as stage II in 17 (15.5\%), stage III in 17 (25.5\%), and stage IVa in 65 (59.1\%) patients. The majority of patients were smokers, nondrinkers with excellent performance status. There were no significant differences between induction and no-induction groups regarding the gender, World Health Organization (WHO) histological subtype, Eastern Cooperative Oncology Group (ECOG) performance status, smoking, and alcohol drinking ( $p>0.05$ for all comparisons). Patients who were younger, EBV-positive, and had advanced-stage group, advanced $T$ and $\mathrm{N}$ categories were more likely to be treated according to induction group compared with no-induction group ( $p<0.05$ for all comparisons). IC was well tolerated compared with AC; within the induction group, 56/65 (86.1\%) patients completed three cycles of IC compared with 13/45 (29\%) patients within the no-induction group. For the induction group, response evaluation after IC revealed that 23/65 (35\%) patients achieved complete response (CR), 37/65 (57\%) patients achieved partial response (PR), and 5/65 (8\%) had stable disease (SD), respectively. On follow-up imaging, 3 months post completion of treatment in both groups, 60/65 (92\%) patients achieved CR within the induction group, while, 44/45 (98\%) patients achieved CR within the no-induction group.

Median follow-up for all patients was 51 months (range: 20-108). A total of nine patients died. Six patients died on follow-up from: a) tumor bleeding $(n=1)$, b) pulmonary 
Table 1 Patient and tumor characteristics

\begin{tabular}{|c|c|c|c|c|}
\hline Characteristic & $\begin{array}{l}\text { Whole cohort } \\
(n=110)\end{array}$ & $\begin{array}{l}\text { Induction } \\
(n=65)\end{array}$ & $\begin{array}{l}\text { No induction } \\
(n=45)\end{array}$ & $\begin{array}{l}\text { Induction versus } \\
\text { no induction } \\
p \text {-Value }\end{array}$ \\
\hline \multicolumn{5}{|c|}{ Age at diagnosis $(\mathrm{y})$} \\
\hline Median (range) & $44(18-76)$ & $37(20-76)$ & $49(18-75)$ & 0.0011 \\
\hline \multicolumn{5}{|l|}{ Gender } \\
\hline Male & 78 (70.9\%) & $45(69.2 \%)$ & $33(73.3 \%)$ & \multirow[t]{2}{*}{0.64136} \\
\hline Female & 32 (29.1\%) & $20(30.8 \%)$ & 12 (26.7\%) & \\
\hline \multicolumn{5}{|l|}{ EBV status } \\
\hline Negative & $34(53.1 \%)$ & $15(41.7 \%)$ & $19(67.9 \%)$ & \multirow[t]{3}{*}{0.03726} \\
\hline Positive & $30(46.9 \%)$ & $21(58.3 \%)$ & $9(32.1 \%)$ & \\
\hline Not tested & 46 & 29 & 17 & \\
\hline \multicolumn{5}{|l|}{ WHO type } \\
\hline 1 & $2(1.8 \%)$ & $2(3.1 \%)$ & & \multirow[t]{3}{*}{0.07926} \\
\hline IIA & $10(9.1 \%)$ & $3(4.6 \%)$ & $7(15.6 \%)$ & \\
\hline IIB & $98(89.1 \%)$ & 60 (92.3\%) & $38(84.4 \%)$ & \\
\hline \multicolumn{5}{|l|}{ Smoking } \\
\hline No & 72 (65.5\%) & $42(64.6 \%)$ & $30(66.7 \%)$ & \multirow[t]{2}{*}{0.82397} \\
\hline Yes & $38(34.5 \%)$ & $23(35.4 \%)$ & $15(33.3 \%)$ & \\
\hline \multicolumn{5}{|l|}{ Drinking } \\
\hline No & 107 (97.3\%) & 64 (98.5\%) & $43(95.6 \%)$ & \multirow[t]{2}{*}{0.35756} \\
\hline Yes & $3(2.7 \%)$ & 1 (1.5\%) & $2(4.4 \%)$ & \\
\hline \multicolumn{5}{|l|}{ ECOG PS } \\
\hline 0 & $98(89.1 \%)$ & $57(87.7 \%)$ & 41 (91.1\%) & \multirow[t]{3}{*}{0.31263} \\
\hline 1 & $11(10.0 \%)$ & $8(12.3 \%)$ & $3(6.7 \%)$ & \\
\hline 2 & $1(0.9 \%)$ & & $1(2.2 \%)$ & \\
\hline \multicolumn{5}{|l|}{ T-category } \\
\hline $\mathrm{T} 1, \mathrm{~T} 2$ & $42(38.2 \%)$ & $13(20 \%)$ & $29(64.4 \%)$ & \multirow[t]{2}{*}{0.0001} \\
\hline $\mathrm{T} 3, \mathrm{~T} 4$ & $68(61.8 \%)$ & $52(80 \%)$ & $16(35.6 \%)$ & \\
\hline \multicolumn{5}{|l|}{ N-category } \\
\hline N0, N1 & $42(38.2 \%)$ & $16(24.6 \%)$ & $26(57.8 \%)$ & \multirow[t]{2}{*}{0.00043} \\
\hline $\mathrm{N} 2, \mathrm{~N} 3$ & $68(61.8 \%)$ & 49 (75.4\%) & $19(42.2 \%)$ & \\
\hline \multicolumn{5}{|c|}{ UICC/AJCC $8^{\text {th }}$ edition stage grouping } \\
\hline$\|$ & $17(15.5 \%)$ & - & $17(37.8 \%)$ & \multirow[t]{3}{*}{0.0001} \\
\hline III & $28(25.5 \%)$ & $13(20.0 \%)$ & $15(33.3 \%)$ & \\
\hline IVA & $65(59.1 \%)$ & $52(80.0 \%)$ & $13(28.9 \%)$ & \\
\hline
\end{tabular}

Note: Values are presented as number (\%) unless otherwise indicated. Significant $P$-values in bold. $P$-values were calculated using a chi-square test. Abbreviation: PS, performance status.

embolism $(n=1)$, c) sepsis $(n=2)$, and d) pneumonia $(n=2)$. While three patients died of unknown causes. The 3-year DFS was $85.33 \%$, whereas the 3-year OS was $92.86 \%$ (-Fig. 1) for the whole cohort. By treatment group (i.e., induction vs. no-induction), there was no significant difference in DFS and OS at 3 years; the 3-year DFS and OS rates were $79.67 \%$ and $92.77 \%$ for the IC group, and $93.28 \%$ and $92.922 \%$ for the no-induction group, respectively ( - Fig. $2 \mathrm{~A}$ and $\mathrm{B})$.
Factors evaluated by univariable analysis are shown in -Table 2. On multivariable analysis, ECOG PS 1 versus 0 was associated with poor OS, (hazard ratio $[\mathrm{HR}]=0.95, \quad 95 \% \quad \mathrm{CI}: \quad 0.10-9.19, \quad p<0.0077)$ (-Table 2).

The estimated 3-year LF, RF, and DF cumulative incidence rates were $58.5 \%$ (95\% CI: 8.4-89\%), 58.00\% (95\% CI: 8-88.8\%), and $63.90 \%$ (95\% CI: $14.1-90.2 \%)$, respectively. Ten patients had LF at the median time of 25 (range: $0-75.9$ ) months; $7 / 10$ 


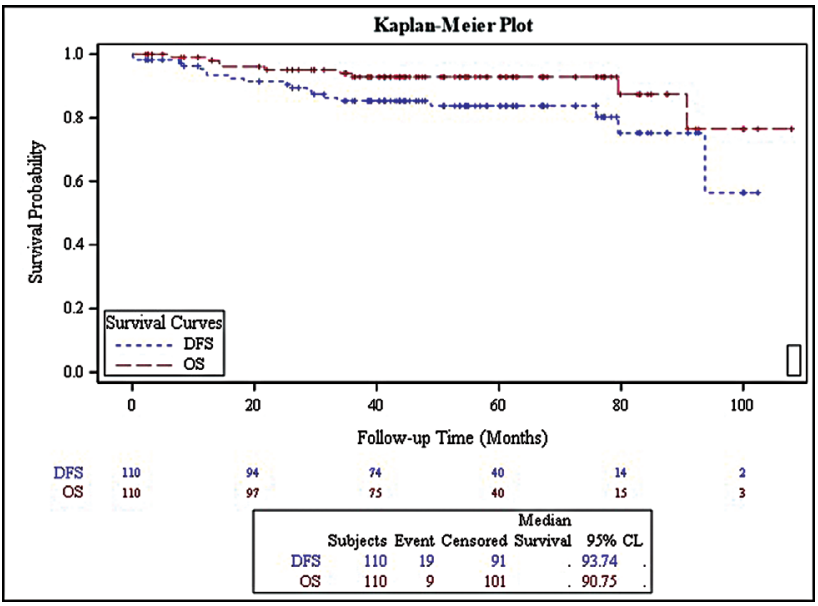

Fig. 1 Kaplan-Meier curve for overall and disease-free survival in the entire study population.

(70\%) in the induction group, and $3 / 10(30 \%)$ in the noinduction group. All patients received palliative chemotherapy. The median time from local recurrence to death was 6 months.

Ten patients developed RF in N1 ( $n=1), \mathrm{N} 2(n=4)$, and N3 disease $(n=5)$, at median 25 months (range: $6-94) ; 6 / 10$ $(60 \%)$ in the induction group and $4 / 10(40 \%)$ in the noinduction group. Six patients who had RF with synchronous LF were treated with palliative chemotherapy. One patient was salvaged with modified radical neck dissection and three had unresectable regional recurrences and were treated with palliative chemotherapy. The median time from regional recurrence to death was 5 months.

Thirteen patients had DM at a median of 18 (range: 6-49) months, $10 / 13(77 \%)$ in the induction group, and $3 / 13(23 \%)$ in the no-induction group. The median time from distant metastasis to death was 6 months.

No grade 4 or 5 acute toxicity was reported. Most of the patients developed grade I or II acute treatment-related toxicities. There was no significant difference in the proportion of acute toxicities between patients who received induction versus no-induction except for more frequent anemia in the induction group $(p<0.01)$.

Late toxicities were mainly graded 1 or 2 . The most common type of late grade II toxicity was xerostomia $69 / 110$ (62.7\%). Four patients had grade I spinal cord toxicity 4/110 (3.6\%), 5/110 (4.5\%) patients had grade II late brain toxicity, and $1(0.9 \%)$ patient had grade I XII cranial nerve palsy. In comparison, between late toxicity in induction and no-induction groups, late brain grades I and II toxicity and late grades II and III esophageal toxicity were more frequent in the induction group compared with the no-induction group with $(p=0.00332)$ and $(0.01049)$, respectively.

\section{Discussion}

In this study, we evaluated and compared clinical outcomes, patterns of failure, and toxicity of locally advanced NPC patients treated with platinum-based CCRT with or without IC or AC at our institution. For the whole cohort, our study revealed survival outcomes comparable to the published literature from the endemic region and favorable to previous studies from our region that used non-IMRT techniques or small series IMRT technique. ${ }^{8,9,11}$ This study did not show a survival advantage for induction over the no-induction group; the 3-year OS and DFS were $92.7 \%$ and $79.67 \%$ in the induction group and $92.9 \%$ and $93.28 \%$ in the no-induction group, respectively. As shown in -Table 1, the majority of patients in the induction group had advanced $\mathrm{T}$ and $\mathrm{N}$ categories compared with the no-induction group. Despite unfavorable prognostic features in the induction group; patients had comparable survival results, which was most likely related to the effect of IC on locoregional control and distant metastasis. We noted a higher incidence of acute anemia among patients treated with induction than among those who were treated with no-induction chemotherapy. The incidence of severe late complications was low in both groups, and we did not find any treatment-related deaths. Interestingly, our patients who were treated with IC had more late brain and esophageal toxicity. This was mainly due to large proportion of patients with T3-4 and N2-3 disease in
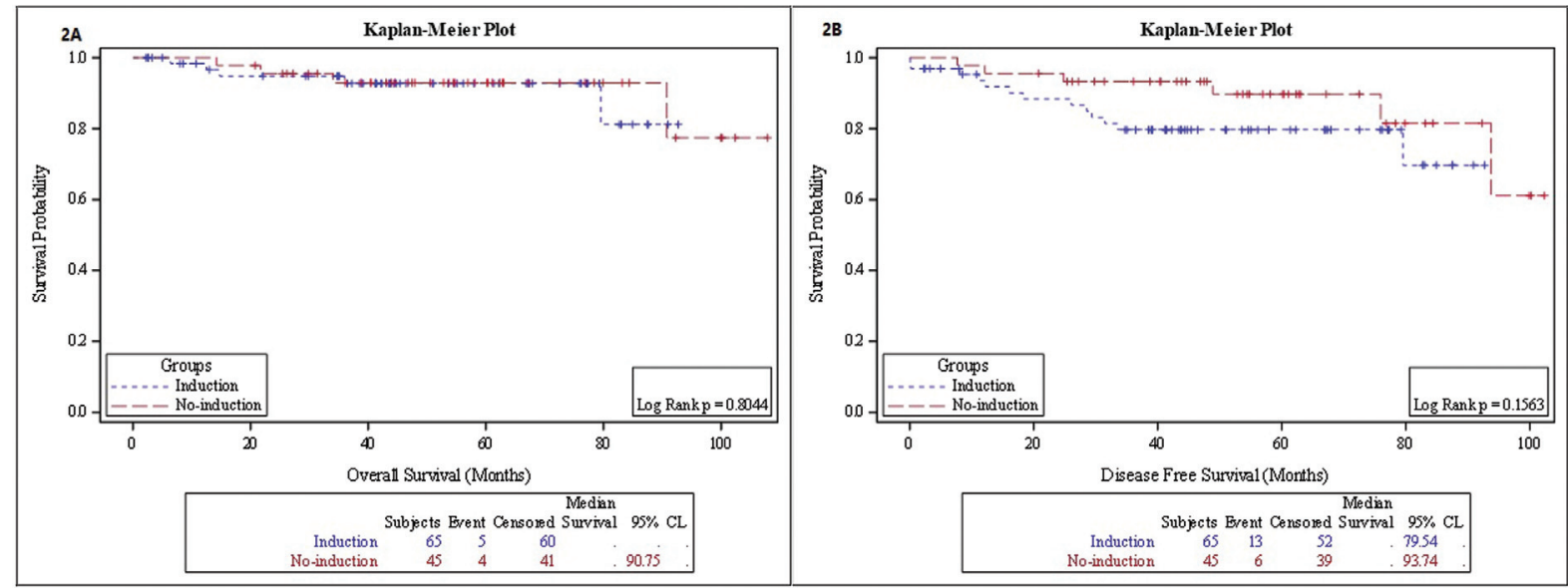

Fig. 2 (A and B) Kaplan-Meier curve comparing overall and disease-free survival according to treatment. 


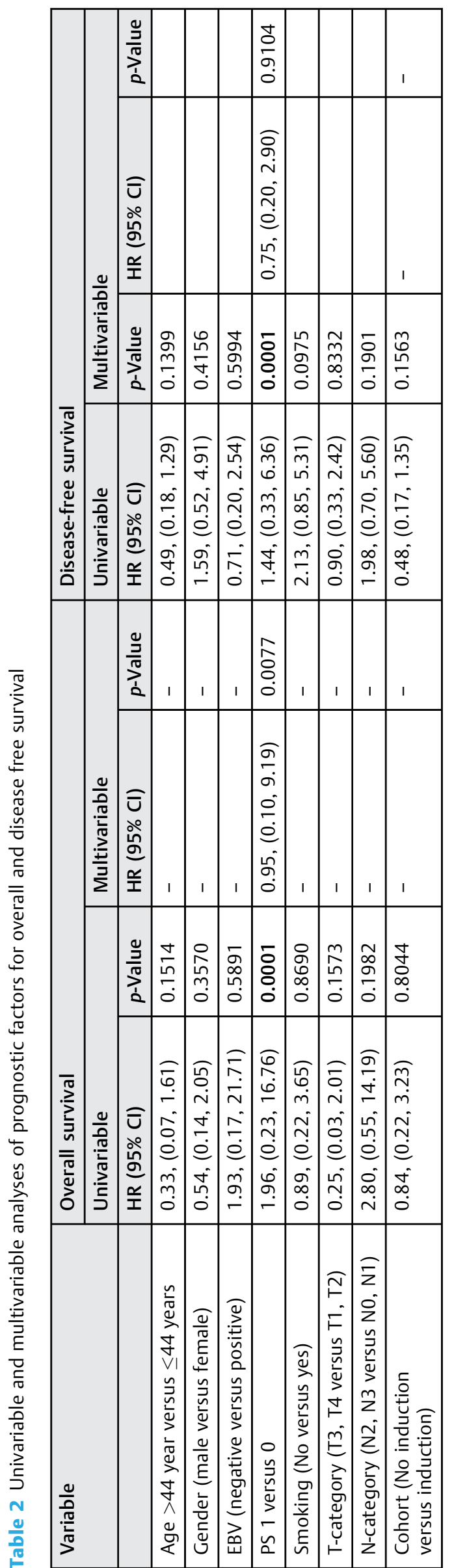

the IC versus CCRT groups (T3-4: $80 \%$ vs. $35 \%, p=0.0001$ and N2-3: $75.4 \%$ vs. $42.2 \%, p=0.0004)$, where irradiating large volume primary tumor (i.e., T3-4) would have an impact on brain toxicity, while extensive nodal involvement (i.e., N2-3) with close proximity to esophagus would result in more frequent late esophageal toxicity.

An overall grade $\geq 2$ late dysphagia was higher in the induction than in the no-induction group but comparable to prior reports. The overall grade $\geq 2$ late toxicity in previous trials ranged from $71 \%$ to $87 \% .^{12,13}$

A recently published randomized phase III clinical trials from the endemic region showed survival advantage favoring IC followed by CCRT, compared with CCRT alone. ${ }^{5}$ Another phase III RCT that showed better 3-year recurrence-free survival, OS, and distant metastasis in the IC group with cisplatin/gemcitabine as compared with the CCRT group. ${ }^{7}$ These two-trial compared IC chemotherapy followed by CCRT to CCRT without additional chemotherapy. One of the systematic reviews showed that IC followed by CCRT did not have a better survival advantage over CCRT with or without additional chemotherapy. ${ }^{14}$ Nonetheless, in spite of these findings, there are still potential advantages to IC beyond the focus on survival or distant metastatic figures.

In our experience, we followed the mainstay treatment of NPC according to the intergroup 0099 RCT when possible, ${ }^{2}$ especially for stage II disease. However, many of our patients presented with advanced local stages where IC was used without jeopardizing long-term outcomes in comparison to the published literature. It is possible that the nonendemic region will inherently have a higher fraction of their patients presenting with advanced stages due to limit access to health care and delays in diagnosis. In this case, there might be a greater role for IC in the developing compared with the developed countries. The local extension of $\mathrm{T} 4$ tumors of our patients also puts a burden on trying to minimize toxicity to vital structures such as the optic chiasm, especially because high rates of longterm survival are expected and are achievable. Another reason to consider IC, that is peculiar to countries with fewer resources, is the availability of IMRT in a timely manner, which can be challenging at times due to the large ratio of patients to radiation machines that we encountered in Jordan.

Our study had some limitations, including retrospective nature and the lack of data on some of EBV status and titer. ${ }^{15}$ Additionally, the sample size was limited due to the rarity of NPC in our region. Furthermore, this study was conducted at a single cancer center from a nonendemic region. Nonetheless, this is the largest retrospective study comparing two standard treatment approaches, predictors of outcomes, patterns of failure and toxicity of advanced NPC in the era of IMRT from our region. Moreover, the selection of IC in large $\mathrm{T} 4$ and N2, N3 diseases has resulted in favorable outcomes comparable to developed countries. However, the challenge remains to improve distance control for NPC.

\section{Conclusions}

Our study showed similar outcomes and patterns of failure between two standard treatment approaches in 
locoregionally advanced NPC. However, multicenter prospective clinical trials are warranted to advance the standard of care of NPC in the Middle East region.

\author{
Ethics Approval \\ Institutional IRB approval was obtained to conduct this \\ study
}

\section{Funding}

None.

\section{Conflict of Interest}

None.

\section{References}

1 Altun M, Fandi A, Dupuis O, Cvitkovic E, Krajina Z, Eschwege F. Undifferentiated nasopharyngeal cancer (UCNT): current diagnostic and therapeutic aspects. Int J Radiat Oncol Biol Phys 1995; 32(03):859-877. Doi: 10.1016/0360-3016(95)00516-2

2 Al-Sarraf M, LeBlanc M, Giri PG, et al. Chemoradiotherapy versus radiotherapy in patients with advanced nasopharyngeal cancer: phase III randomized Intergroup study 0099. J Clin Oncol 1998;16 (04):1310-1317. Doi: 10.1200/JCO.1998.16.4.1310

3 Forastiere AA, Shank D, Neuberg D, Taylor SG IV, DeConti RC, Adams G. Final report of a phase II evaluation of paclitaxel in patients with advanced squamous cell carcinoma of the head and neck: an Eastern Cooperative Oncology Group trial (PA390). Cancer 1998;82(11):2270-2274

4 Chan AT, Leung SF, Ngan RK, et al. Overall survival after concurrent cisplatin-radiotherapy compared with radiotherapy alone in locoregionally advanced nasopharyngeal carcinoma. J Natl Cancer Inst 2005;97(07):536-539. Doi: 10.1093/jnci/dji084

5 Chen L, Hu CS, Chen XZ, et al. Concurrent chemoradiotherapy plus adjuvant chemotherapy versus concurrent chemoradiotherapy alone in patients with locoregionally advanced nasopharyngeal carcinoma: a phase 3 multicentre randomised controlled trial. Lancet Oncol 2012;13(02):163-171. Doi: 10.1016/S1470-2045 (11)70320-5

6 Sun Y, Li WF, Chen NY, et al. Induction chemotherapy plus concurrent chemoradiotherapy versus concurrent chemoradiotherapy alone in locoregionally advanced nasopharyngeal carcinoma: a phase 3, multicentre, randomised controlled trial. Lancet Oncol 2016;17(11):1509-1520. Doi: 10.1016/S1470-2045 (16)30410-7

7 Zang J, Xu M, Li C, et al. Gemcitabine and cisplatin versus docetaxel and cisplatin as induction chemotherapy followed by concurrent chemoradiotherapy in locoregionally advanced nasopharyngeal carcinoma from non-endemic area of China. J Cancer Res Clin Oncol 2020;146(09):2369-2378. Doi: 10.1007/s00432-02003229-3

8 Maklad AM, Bayoumi Y, Senosy Hassan MA, et al. Patterns of failure and survival in patients with nasopharyngeal carcinoma treated with intensity-modulated radiation therapy in Saudi Arabia. OncoTargets Ther 2016;9:6561-6567. Doi: 10.2147/OTT. S95457

9 Mohamad I, Salem A, Abu Hejleh T, et al. Peer-assisted learning: intensity-modulated radiotherapy transition in developing countries. Clin Oncol (R Coll Radiol) 2017;29(10):689-695. Doi: 10.1016/j.clon.2017.06.002

10 Amin MB, Greene FL, Edge SB, et al. The eighth edition AJCC Cancer Staging Manual: continuing to build a bridge from a populationbased to a more "personalized" approach to cancer staging. CA Cancer J Clin 2017;67(02):93-99. Doi: 10.3322/caac.21388

11 Kam MK, Teo PM, Chau RM, et al. Treatment of nasopharyngeal carcinoma with intensity-modulated radiotherapy: the Hong Kong experience. Int J Radiat Oncol Biol Phys 2004;60(05): 1440-1450. Doi: 10.1016/j.ijrobp.2004.05.022

12 Au KH, Ngan RKC, Ng AWY, et al. Treatment outcomes of nasopharyngeal carcinoma in modern era after intensity modulated radiotherapy (IMRT) in Hong Kong: A report of 3328 patients (HKNPCSG 1301 study). Oral Oncol 2018;77:16-21. Doi: 10.1016/ j.oraloncology.2017.12.004

13 Chen YP, Tang LL, Yang $\mathrm{Q}$ et al. Induction chemotherapy plus concurrent chemoradiotherapy in endemic nasopharyngeal carcinoma: individual patient data pooled analysis of four randomized trials. Clin Cancer Res 2018;24(08):1824-1833. Doi: 10.1158/1078-0432.CCR-17-2656

14 Ribassin-Majed L, Marguet S, Lee AWM, et al. What is the best treatment of locally advanced nasopharyngeal carcinoma? An individual patient data network meta-analysis. J Clin Oncol 2017; 35(05):498-505. Doi: 10.1200/JCO.2016.67.4119

15 Wang WY, Twu CW, Chen $\mathrm{HH}$, et al. Long-term survival analysis of nasopharyngeal carcinoma by plasma EpsteinBarr virus DNA levels. Cancer 2013;119(05):963-970. Doi: $10.1002 /$ cncr.27853 